\title{
Prospective, Multicentre Trial of Methoxyflurane for Acute Trauma-Related Pain in Helicopter Emergency Medical Systems and Hostile Environments: METEORA Protocol
}

\author{
Franco Marinangeli - Giorgio Reggiardo - Antonella Sblendido • \\ Amedeo Soldi · Alberto Farina · On behalf of The METEORA Group
}

Received: September 19, 2018 / Published online: October 29, 2018

(C) The Author(s) 2018

\begin{abstract}
Introduction: The inhalational analgesic lowdose methoxyflurane has been widely used by Australian ambulance services since 1975 and is now approved in Europe for emergency relief of moderate-to-severe trauma-related pain in conscious adult patients. The use of methoxyflurane in hostile environments is of special interest given its portability, ease of use and rapid onset of action. This trial will investigate the efficacy, tolerability and practicality of use of inhaled methoxyflurane in patients with moderate-to-severe trauma-related pain rescued from hostile mountainous environments by the Helicopter Emergency Medical Service (HEMS) in Italy.
\end{abstract}

Enhanced digital features To view enhanced digital features for this article go to https://doi.org/10.6084/ m9.figshare.7165537.

Electronic supplementary material The online version of this article (https://doi.org/10.1007/s12325018-0816-8) contains supplementary material, which is available to authorized users.

F. Marinangeli

Department of Anesthesiology and Intensive Care, University of L'Aquila, L'Aquila, Italy

G. Reggiardo

Medi Service srl, Genoa, Italy

A. Sblendido $\cdot$ A. Soldi $\cdot$ A. Farina $(\bowtie)$

Mundipharma Pharmaceuticals srl, Milan, Italy

e-mail: alberto.farina@mundipharma.it
Methods: METEORA is a phase IIIb, prospective, single-arm, multicentre trial. Approximately 200 adult patients with a pain score of at least 4 on the numerical rating scale (NRS) due to limb trauma rescued by HEMS will be enrolled. Patients will receive up to $2 \times 3 \mathrm{~mL}$ methoxyflurane, self-administered by the patient by inhalation under medical supervision. Rescue medication will be permitted if required.

Planned Outcomes: Pain intensity will be measured using a 100-mm visual analogue scale (VAS) at baseline, at 5, 10, 15, 20, 30, 45 and $60 \mathrm{~min}$ after the start of methoxyflurane inhalation and when positioning the patient on a spinal board or stretcher; and also using the NRS at enrolment and at $10 \mathrm{~min}$. Use of rescue medication (yes/no) will be recorded. The patient will rate efficacy and the healthcare professional will rate practicality of methoxyflurane treatment at 30 and $60 \mathrm{~min}$ using a 5-point Likert scale. Vital signs will be measured at baseline, 10, 30 and $60 \mathrm{~min}$. Assessments after $30 \mathrm{~min}$ will only be performed for patients using a second inhaler. Adverse events will be recorded until safety follow-up at $3 \pm 1$ days. The primary endpoint is the percentage of patients achieving at least $30 \%$ improvement from baseline in VAS pain intensity within the first $10 \mathrm{~min}$ of methoxyflurane administration.

Trial Registration: EudraCT number: 2017-004601-40. 
Funding: Mundipharma Pharmaceuticals, srl.

Plain Language Summary: Plain language summary available for this article.

\section{PLAIN LANGUAGE SUMMARY}

The treatment of pain is an essential part of the management of injured patients. In emergency rescue situations, rapid and effective pain relief can reduce the patient's stress and discomfort, making it easier to assess, treat and extricate them. Currently available painkillers have limitations such as being slow to work (oral medications), requiring needles (intravenous medications) or prolonged monitoring and observation (e.g. opioids). An inhaled painkiller (methoxyflurane) is now available in Europe for emergency relief of moderate-to-severe pain in conscious adult patients with trauma (injury) and associated pain. Methoxyflurane is administered via a hand-held inhaler, which provides pain relief within 6-10 inhalations and lasts for 25-30 min, on average, when used continuously. The patient can control his/her own level of pain relief and a second inhaler may be used if required. Methoxyflurane has been widely used by Australian ambulance services since 1975 and its effectiveness and safety are well established. Considering its ease of use and rapid action, inhaled methoxyflurane may be useful in emergency situations in remote and hostile environments. A new trial (METEORA) will assess the use of methoxyflurane in 200 patients with limb injuries who are rescued from mountainous environments by the Helicopter Emergency Medical Service (HEMS) in Italy. Patients with moderate-to-severe pain will receive inhaled methoxyflurane under medical supervision. A second inhaler and/or additional pain-relieving medication will be provided if necessary. The trial will assess the reduction in pain intensity and whether additional pain-relieving medication is needed. The practicality of use of methoxyflurane in the emergency rescue situation and any side effects will also be evaluated.
Keywords: Acute pain; Analgesic; HEMS (Helicopter Emergency Medical Service); Inhaled analgesic; Methoxyflurane; Penthrox; Prehospital; Trauma

\section{INTRODUCTION}

The treatment of pain is important in the management of trauma patients, both as a fundamental human right and because intense pain can cause clinical deterioration [1]. Numerous studies have demonstrated that inadequate pain treatment can lead to cardiovascular, respiratory, gastrointestinal, urological and infection complications [2]. In emergency rescue situations, effective analgesia reduces the patient's physiological and psychological stress and aids extrication and procedural interventions such as realignment and splinting of fractures. However, the undertreatment of acute pain ("oligoanalgesia") in the pre-hospital emergency setting remains a significant problem $[2,3]$. Albrecht and colleagues reported a prevalence of oligoanalgesia of $43 \%$ in a 10 -year retrospective study of adult trauma patients transported by a physician-staffed air-medical transport system in Switzerland [4]. More recently, Oberholzer and coworkers reported an incidence of $38 \%$ in a similar setting [5].

In Europe, commonly used analgesics for trauma pain include paracetamol, non-steroidal anti-inflammatory drugs, nitrous oxide, ketamine and opioids [3, 6-8]. Besides analgesic efficacy, safety profile and ease of use will influence the treatment choice, particularly in emergency situations in remote and/or hostile environments where cannulation may be difficult or where bulky equipment cannot be carried. Opioids are generally administered intravenously and require continuous physiological monitoring during use due to their challenging safety profile. Ketamine is a nonopioid option used by some helicopter emergency services [8], but is usually administered intravenously, causes sedation and is mostly used as an adjuvant to opioid analgesics for severe pain [9]. Fentanyl (by intranasal or oral transmucosal route) or intranasal ketamine may 
be an analgesic option but are not suitable for patients with facial trauma. Transmucosal fentanyl is only indicated for breakthrough cancer pain, and not traumatic pain, in many countries. Nitrous oxide requires heavy, bulky cylinders and is contraindicated in any condition where gas is entrapped within the body and where expansion might be dangerous (e.g. pneumothorax, air embolism, pneumocephalus) [10]. According to the Italian Intersociety Recommendations (SIAARTI, SIMEU, SIS 118, AISD, SIARED, SICUT, IRC) on emergency pain management, "The ideal pre-hospital analgesic should be simple to use, safe, effective, unaffected by transport times, have a rapid onset, a short duration of action, and able to be titrated to achieve the desired effect in all patients" [11]. These ideal characteristics could be even more relevant in the helicopter rescue of a traumatized patient from a hostile environment.

The inhalational analgesic low-dose methoxyflurane has been used extensively by Australian ambulance and air ambulance services and emergency departments since the 1970s and has recently been licensed in Europe for emergency relief of moderate-to-severe pain in conscious adult patients with trauma and associated pain [12]. Low-dose methoxyflurane is a non-narcotic agent that is self-administered by the patient via a lightweight, disposable, hand-held inhaler (Penthrox ${ }^{\circledR}$, Medical Developments International Limited, Scoresby Medical, Australia) under the supervision of a trained person, with a maximum dose of two 3-mL vials in a 24-h period [12]. Low-dose methoxyflurane has been shown to provide effective short-term pain relief in both adults and children in emergency medicine [13-20] and minor surgical and dental procedures [21-28]. Methoxyflurane's rapid onset (within 4-5 min $[15,16]$ ) and proven stability over a wide temperature range ( -20 to $40{ }^{\circ} \mathrm{C}$ ), combined with its portability and ease of use, make it a suitable candidate for analgesia in emergency situations in hostile environments [29].

Although there are many studies published in the scientific literature that support the efficacy and safety of methoxyflurane for analgesic use in both adult and paediatric patients, few randomised controlled trials (RCTs) have been conducted. The STOP! study was a UK-based RCT that demonstrated significantly greater reductions in pain intensity, median time to pain relief of $4 \mathrm{~min}$, along with significantly less use of rescue medication and high patient satisfaction in patients treated with methoxyflurane compared with placebo [15], but has the limitation of being placebo- and not activecontrolled. A prospective RCT is currently underway to assess the efficacy and rapidity of the analgesic effect of inhaled methoxyflurane compared with standard of care in the treatment of moderate-to-severe trauma-related pain in the pre-hospital (ambulance) and emergency department setting (MEDITA study, EudraCT 2017-001565-25 [30]). The study will expand the efficacy data available for methoxyflurane from RCTs. The use of methoxyflurane in hostile environments, where mobilization of the patient is difficult, is of special interest given its ease of use and rapid onset of action. Therefore, a second trial, which we describe here, has been launched to assess the efficacy, tolerability and practicality of use of inhaled methoxyflurane in the treatment of moderate-to-severe trauma-related pain in this specific setting.

\section{METHODS}

\section{Trial Design}

The trial is a phase IIIb, prospective, single-arm, open-label, multicentre clinical evaluation of inhaled methoxyflurane for the emergency treatment of moderate-to-severe trauma-related pain in patients rescued from hostile mountainous environments by the Helicopter Emergency Medical Service (HEMS) (METEORA; study code MR311-3505; EudraCT 2017-004601-40). The study is being conducted at approximately 11 helicopter rescue bases belonging to the HEMS association in Italy (i.e. in the pre-hospital setting). HEMS bases were chosen according to the geographical features of the areas of intervention (mountain bases) and possibility of using a rescue winch [31].

Conscious adult, stable, alert and co-operative patients with minor trauma to the limbs 
(fracture, dislocation, luxation, crushing or contusion) in a single area and a pain score of at least 4 on the 11-point numerical rating scale (NRS) who are rescued via the HEMS will be assessed for eligibility in the trial. Full inclusion and exclusion criteria are presented in Table 1. It is planned to enrol a total of 200 patients. Patients will receive up to two $3-\mathrm{mL}$ inhaled methoxyflurane vials and undergo trial assessments in a single day (the day of enrolment), with a follow-up telephone call $3 \pm 1$ days later to assess safety and record any concomitant therapies.

\section{Trial Treatment}

After eligibility is confirmed and baseline assessments have been completed, patients will receive one hand-held inhaler with a $3-\mathrm{mL}$ vial of methoxyflurane (Fig. 1). Prior to use, the methoxyflurane liquid is added to the inhaler via a one-way valve and is absorbed by a polypropylene wick; once absorbed, the liquid

Table 1 Patient eligibility criteria

Inclusion criteria

Age $\geq 18$ years

Stable, alert and co-operative patient, i.e. able to understand and communicate with the investigator in order to perform the trial activities

Minor trauma to the limbs (fracture, dislocation, luxation, crushing or contusion) in a single area

Note: given the setting, confirmation by means of diagnostic tests and procedures is not required for recruitment; the suspicion of involvement of a single area is sufficient

Moderate-to-severe pain (numerical rating scale score $\geq 4$ ) secondary to minor trauma

Written informed consent should be given by each patient before any trial-specific activity. In cases where the patient is not able to write autonomously, verbal consent must be obtained in the presence of an impartial witness, and the patient will be required to confirm independently as soon as he/she is able

Exclusion criteria

Hypersensitivity to methoxyflurane, to any fluorinated anaesthetic or to the excipient butylated hydroxytoluene E321

Personal or family history (parents or siblings) of malignant hyperthermia

History of serious adverse reactions to inhalation anaesthetics

History of renal or liver failure

Clinically evident cardiovascular instability

Clinically evident respiratory depression

Lactation and known or suspected pregnancy as reported by the patient

Note: a delay of even 1 day from the scheduled date of menstruation ( 28 days from the beginning of the last menstruation) is considered as a suspected pregnancy

Ongoing treatment with any analgesic for chronic pain or such treatment within the previous $8 \mathrm{~h}$

Acute intoxication due to medications, drugs or alcohol

Imminent risk to life requiring hospitalisation in the operating room or resuscitation

Altered level of alertness and/or consciousness (Glasgow coma scale [GCS] < 15) 


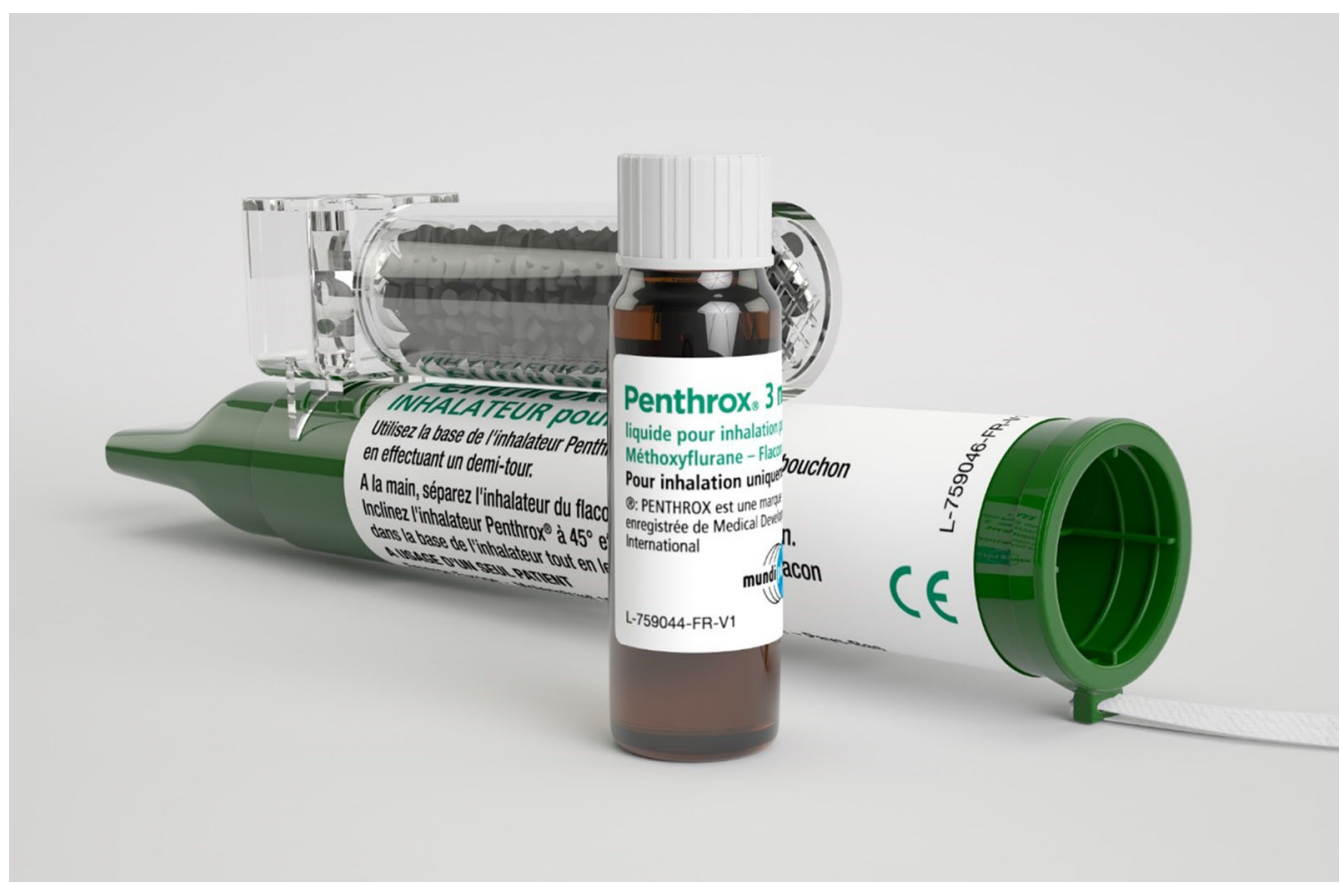

Fig. 1 Methoxyflurane inhaler with activated carbon chamber

vaporizes and the patient inhales the vapour through the mouthpiece. The patient also exhales back into the mouthpiece, so that any exhaled methoxyflurane is captured by the activated charcoal (AC) chamber, which adsorbs methoxyflurane and prevents fugitive emissions resulting in occupational exposure. The patient will self-administer methoxyflurane via inhalation under the supervision of trained healthcare personnel. Patients will be instructed to breathe intermittently to obtain adequate analgesia. One inhaler ( $3 \mathrm{~mL}$ methoxyflurane) will provide approximately $1 \mathrm{~h}$ of analgesia under suggested intermittent inhalation conditions; continuous inhalation reduces the duration of use to approximately $25 \mathrm{~min}$ of analgesia. A second inhaler will be provided if requested by the patient, but no patient will receive a dose greater than $6 \mathrm{~mL}$ methoxyflurane. All patients enrolled into the trial will receive the same trial treatment (up to $6 \mathrm{~mL}$ inhaled methoxyflurane) regardless of pain severity.

If a patient experiences insufficient analgesia, rescue medication will be permitted from 25 min after the start of inhalation and measurement of pain relief for this time point.
However, the investigator may administer rescue medication at any time at his/her discretion. Rescue medication will be chosen and administered according to local clinical practice and recorded in the case report form (CRF).

\section{Trial Objectives and Endpoints}

The primary objective of the trial is to assess the efficacy of inhaled methoxyflurane in the treatment of moderate-to-severe acute traumarelated pain in patients rescued in a hostile environment. The objectives of the trial and associated endpoints are presented in Table 2. Pain intensity will be assessed using a $100-\mathrm{mm}$ visual analogue scale (VAS; $0=$ no pain to $100=$ maximum pain) at baseline (immediately before the start of treatment) and at 5, 10, 15, $20,30,45$ and $60 \mathrm{~min}$ after the start of methoxyflurane inhalation or until rescue medication is administered. The assessments of VAS pain intensity at 45 and 60 min will only be performed for patients who request a second methoxyflurane inhaler. VAS pain intensity will also be assessed when positioning the patient on a spinal board or stretcher. The patient will 
Table 2 Trial objectives and endpoints

Objective Endpoint

Primary objective

To demonstrate that inhaled methoxyflurane is effective within $10 \mathrm{~min}$ in the treatment of moderate-to-severe acute trauma pain

Secondary objectives

To assess the efficacy of inhaled methoxyflurane in the treatment of moderate-to-severe acute trauma pain (after $5,10,15,20,30,45$ and $60 \mathrm{~min}$ )

To assess the efficacy of inhaled methoxyflurane in the treatment of moderate-to-severe acute trauma pain in terms of the use of additional analgesia (rescue medication)

To assess the efficacy of inhaled methoxyflurane in the opinion of the patient and the practicality of using the device in the opinion of the healthcare provider

To assess the safety and tolerability of inhaled methoxyflurane
Percentage of patients achieving $\geq 30 \%$ improvement from baseline in VAS pain intensity within the first $10 \mathrm{~min}$ of methoxyflurane administration

Change in pain intensity as measured using the VAS from baseline to up to $10 \mathrm{~min}$ after the start of methoxyflurane administration

Percentage of patients achieving $\geq 30 \%$ improvement from baseline in VAS pain intensity and an NRS score of 0-3 within the first 10 min of methoxyflurane administration

Change in VAS pain intensity at 5, 10, 15, 20 and $30 \mathrm{~min}$ after the start of methoxyflurane administration, and at 45 and $60 \mathrm{~min}$ if using a second methoxyflurane inhaler

Percentage of patients achieving $\geq 50 \%$ improvement from baseline in VAS pain intensity within the first $10 \mathrm{~min}$ of methoxyflurane administration

Intensity of pain as measured using the VAS when positioning the patient on a spinal board or stretcher

Percentage of patients using a second methoxyflurane inhaler

Percentage of patients resorting to additional analgesia (rescue medication) within $30 \mathrm{~min}$ after the start of methoxyflurane administration

Satisfaction with efficacy of methoxyflurane treatment, assessed by the patient using a 5 -point Likert scale at $30 \mathrm{~min}$ (and $60 \mathrm{~min}$ if using a second inhaler)

Satisfaction with practicality of methoxyflurane, assessed by the healthcare provider using a 5-point Likert scale at $30 \mathrm{~min}$ (and $60 \mathrm{~min}$ if the patient uses a second inhaler)

Incidence of adverse events

Progress of vital signs measured at 10 and $30 \mathrm{~min}$ after baseline measurement (and at $60 \mathrm{~min}$ if the patient uses a second methoxyflurane inhaler) 
Table 2 continued

\begin{tabular}{ll}
\hline Objective & Endpoint \\
\hline $\begin{array}{l}\text { Exploratory objectives } \\
\begin{array}{l}\text { To assess the efficacy of inhaled methoxyflurane with } \\
\text { respect to the type of trauma (fracture, dislocation, } \\
\text { luxation, crushing, contusion) }\end{array}\end{array}$\begin{tabular}{l}
$\begin{array}{l}\text { Efficacy of inhaled methoxyflurane with respect to the type } \\
\text { of trauma (fracture, dislocation, luxation, crushing, } \\
\text { contusion) }\end{array}$ \\
\hline
\end{tabular}
\end{tabular}

$N R S$ numerical rating scale, $V A S$ visual analogue scale

be asked to respond to the question "How much pain do you feel at this moment?" by marking a vertical line on the paper VAS that best represents their state. The VAS is designed to be selfadministered but given the emergency rescue setting and the short detection times for the variable, a specially trained healthcare professional may assist the patient to complete the VAS if required. Pain intensity will also be measured on the NRS scale at enrolment and $10 \mathrm{~min}$ after the start of methoxyflurane inhalation. Whether patients receive a second methoxyflurane inhaler or additional analgesia (rescue medication) will be recorded.

At $30 \mathrm{~min}$ after the start of methoxyflurane inhalation, the patient will be asked to rate the overall efficacy of methoxyflurane treatment by answering the question "Altogether, how do you rate the efficacy of the pain therapy that was given to you?" At the same time point, the healthcare worker (investigator) who has administered the trial treatment will rate the practicality of using methoxyflurane by answering the question "Altogether, how do you rate the practicality of the pain therapy you have given?" Each will be rated on a 5-point Likert qualitative scale ("poor", "fair", "good", "very good" or "excellent"). The same assessments will be repeated at $60 \mathrm{~min}$ if the patient uses a second methoxyflurane inhaler.

Adverse events (not related to the trauma presentation) will be recorded from the start of treatment until the safety follow-up telephone call $3 \pm 1$ days later. Vital signs (supine systolic and diastolic blood pressure, heart rate and respiration rate) will be measured at enrolment and at 10, 30 and $60 \mathrm{~min}$ after the start of methoxyflurane inhalation (60 min vital signs assessment is only required if the patient uses a second methoxyflurane inhaler). Concomitant therapies taken by the patient within 7 days prior to inclusion in the trial and during the following 3 days, including rescue medication use, will be recorded. Additionally, the final diagnosis of the trauma category (fracture, dislocation, luxation, crushing, contusion) will be recorded at the end of the intervention or during the safety follow-up telephone call. All data collected during the trial will be recorded on a paper CRF.

\section{Sample Size Estimate}

The primary objective will be evaluated on the basis of the change from baseline in VAS pain intensity within the first $10 \mathrm{~min}$ of methoxyflurane administration. Assuming a responder rate (patients achieving $\geq 30 \%$ improvement from baseline in VAS pain intensity) under the null hypothesis with $H_{0}$ equal to $70 \%$ and a significance level of 0.05 (two-tailed test), a sample size of 180 evaluable patients will provide $82 \%$ power to detect a difference $\left(P_{1}-\right.$ $P_{0}$ ) of -0.10 . Allowing for $10 \%$ of patients being non-evaluable, it is planned to enrol a total of 200 patients into the trial.

\section{Statistical Analyses}

All trial data will be summarised using descriptive statistics. For quantitative variables, this will include the number of patients evaluated, mean, standard deviation, minimum, maximum and distribution quartiles. For qualitative 
variables, this will include absolute frequencies and percentages at each time point.

The primary population for efficacy analyses will be the intention-to-treat (ITT) population (defined as all patients who receive methoxyflurane and have at least one postbaseline assessment of VAS pain intensity). Confirmatory analyses will be performed on the per-protocol population (defined as all patients in the ITT population without any major protocol violations). The primary endpoint is the percentage of patients achieving at least 30\% improvement from baseline in VAS pain intensity within the first $10 \mathrm{~min}$ of methoxyflurane administration. The reduction in VAS pain intensity from baseline to $10 \mathrm{~min}$ after the start of methoxyflurane administration will also be assessed by means of an inferential comparison; a paired sample $t$ test is proposed for this. The test will calculate the difference between the VAS pain intensity scores at the two time points for each patient and test if the mean is different from 0 . The reported test result will indicate the correlation index between the two time points, the difference between the means, the $t$ test and the $95 \%$ confidence interval of the difference between the means. Changes in VAS pain intensity from baseline to $5,10,15,20,30,45$ and 60 min will be analysed using a generalised linear model for repeated measures including trauma category as a factor and age and baseline NRS score as covariates.

Safety parameters will be summarised for the safety population (defined as patients who receive at least one dose of methoxyflurane). Adverse events will be coded using the Medical Dictionary for Regulatory Activities and summarised by system organ class and preferred term.

\section{STRENGTHS AND LIMITATIONS OF THE STUDY}

The portability and ease of use of methoxyflurane combined with its rapid onset of action (within 6-10 inhalations [15]) and stability over a wide temperature range make it an ideal candidate for emergency analgesia in trauma patients rescued in hostile environments by
HEMS. Considering its fast offset of action (within 3-20 min) and lack of interaction with other agents [12], it may also be employed as a useful bridging analgesic prior to intravenous opioid administration or further medical assessment.

A clear limitation of the trial is that it is open, single-arm and non-comparative. However, it would be logistically challenging and unethical to perform a blinded comparative study in helicopter emergency situations where time and staff are limited and rapid analgesia is vital to make trauma patients more comfortable and aid extrication. Furthermore, methoxyflurane has a unique mode of action and characteristic smell, which makes blinding of treatment difficult. Nevertheless, the trial should still provide valuable insight into the effectiveness, safety and practicality of methoxyflurane analgesia in HEMS and hostile environments.

Risks to the patient receiving methoxyflurane are considered to be minimal. With over 40 years of clinical use as an analgesic in Australia and New Zealand and over 5 million administrations to date, methoxyflurane has an established safety profile. The most common adverse events are related to the central nervous system (e.g. headache, dizziness and somnolence) and are generally mild and transient, resolving after inhalation is stopped [12]. In total, less than 20 adverse event reports have been submitted to the Australian regulatory authorities since methoxyflurane became available as a 3-mL dose for analgesic use [32]. Although nephrotoxicity was previously a concern with much higher anaesthetic doses of methoxyflurane [33], clinical experience suggests that a low but effective analgesic dose within permitted limits is not associated with the risk of renal adverse events [15, 16, 34], and a large data-linkage study including 17,629 patients administered low-dose methoxyflurane during ambulance transport [35] supports the conclusion that low-dose methoxyflurane is not associated with an increased risk of renal disease. Laboratory evidence shows a large safety margin of at least 2.7- to 8-fold for analgesic use of methoxyflurane based on minimum alveolar concentration (MAC)-hours or serum fluoride 
concentrations [34], and at least 10-fold based on occupational methoxyflurane exposure levels without the AC chamber [36]. The AC chamber adsorbs methoxyflurane to reduce occupational exposure by more than $80 \%$ and is mandatory for use across Europe. Analgesic methoxyflurane has no clinically significant effects on cardiovascular or respiratory parameters or consciousness levels [37, 38]. Low-dose methoxyflurane is contraindicated in patients hypersensitive to the agent or to any fluorinated anaesthetics, patients at risk for malignant hyperthermia, patients showing signs of liver damage after previous methoxyflurane use or halogenated hydrocarbon anaesthesia, and those with clinically significant renal impairment, an altered level of consciousness, clinically evident cardiovascular instability or with clinically evident respiratory depression [12].

Two unidimensional pain scales (the 11-point NRS and the 100-mm VAS) will be used for assessment of pain intensity in this study. The NRS score is the most frequently used clinical and emergency tool for pain surveys owing to the ease with which the measurement can be administered and collected [39], and many of the guidelines on the use of pain therapy are based on the NRS score and the related classification (NRS $1-3=$ mild pain; NRS 4-6 = moderate pain; NRS $\geq 7=$ severe pain) $[11,40]$. Therefore, the NRS will be used for the purposes of verifying trial eligibility and will also be used as a secondary efficacy measure. The $100-\mathrm{mm}$ pain VAS is a more sensitive tool (allowing the patient to mark a point on the scale that is measured to the nearest millimetre) and will be used as the primary measure for assessing pain intensity in this trial. The pain VAS is frequently used in pain studies because it is easy to use, requires no verbal or reading skills and is sufficiently versatile to be employed in a variety of settings [41, 42]. The NRS and VAS are valid and reliable for clinical use [43] and have been shown to correspond well [39]. Both scales are designed to be completed by the patient themselves; however, considering the emergency conditions and possible immobility of the patient, a specially trained healthcare worker may assist the patient in completing the scales in this trial.

\section{ETHICS AND DISSEMINATION}

The METEORA trial is sponsored by Mundipharma Research Limited and will be conducted in accordance with International Council on Harmonization Good Clinical Practice adhering to the ethical principles of the Declaration of Helsinki (1964 and subsequent amendments), as well as national and local guidelines. The trial has been approved by the Italian Medicines Agency and is registered with the European Union Clinical Trials Register (EudraCT number 2017-004601-40). All trial documents and procedures will be reviewed and approved by the appropriate ethics committees at each site. Written informed consent will be obtained from all patients before initiation into the trial. Given the emergency setting and the requirement for rapid analgesia, where the patient is unable to provide written informed consent, witnessed verbal consent will be obtained with the patient signing the informed consent as soon as they are able.

\section{CONCLUSION}

Low-dose methoxyflurane has been shown to provide rapid analgesia in the emergency setting and has a well-established safety profile. Given its ease of administration and portability, inhaled low-dose methoxyflurane may offer advantages over other analgesic options in emergency situations in remote and hostile environments. It is anticipated that the results of this study in Italian HEMS, along with findings of other recent and ongoing studies [30, 44, 45], will provide further clinical evidence to support the use of methoxyflurane in this setting.

\section{ACKNOWLEDGEMENTS}

The investigators of the METEORA Group are listed in the Supplementary Materials. ${ }^{\circledR}$ Penthrox is a registered trade mark of Medical Developments International Limited and used under license. 
Funding. This trial is funded by Mundipharma Research Limited. Costs for article processing and Open Access were funded by Mundipharma Pharmaceuticals srl.

Editorial Assistance. Editorial assistance in the preparation of this manuscript was provided by Karen Mower of Scientific Editorial. Support for this assistance was funded by Mundipharma Research Limited.

Authorship. All authors had access to the protocol and Statistical Analysis Plan. All named authors meet the International Committee of Medical Journal Editors (ICMJE) criteria for authorship for this manuscript, take responsibility for the integrity of the work as a whole, and have given final approval to the version to be published.

Disclosures. Franco Marinangeli has nothing to disclose. Giorgio Reggiardo is an employee of the clinical research organization conducting the study. Antonella Sblendido is an employee of Mundipharma Pharmaceuticals srl. Amedeo Soldi is an employee of Mundipharma Pharmaceuticals srl. Alberto Farina is an employee of Mundipharma Pharmaceuticals srl.

Compliance with Ethics Guidelines. The trial will be conducted in accordance with the principles established in the Declaration of Helsinki and the International Conference on Harmonization guidelines for good clinical practice. All trial documents and procedures will be approved by the appropriate ethics committees at each site, and written informed consent (or verbal witnessed consent) will be obtained from all patients before initiation into the study.

Data Availability. Data sharing is not applicable to this article as no datasets have been generated or analyzed during the current study.

Open Access. This article is distributed under the terms of the Creative Commons Attribution-NonCommercial 4.0 International License (http://creativecommons.org/licenses/ by-nc/4.0/), which permits any noncommercial use, distribution, and reproduction in any medium, provided you give appropriate credit to the original author(s) and the source, provide a link to the Creative Commons license, and indicate if changes were made.

\section{REFERENCES}

1. Elsensohn F. Consensus guidelines on mountain emergency medicine and risk reduction. Lecco: Casa Editrice Stefanoni; 2001.

2. Marinangeli F, Narducci C, Ursini ML, et al. Acute pain and availability of analgesia in the prehospital emergency setting in Italy: a problem to be solved. Pain Pract. 2009;9:282-8.

3. Mura P, Serra E, Marinangeli F, et al. Prospective study on prevalence, intensity, type, and therapy of acute pain in a second-level urban emergency department. J Pain Res. 2017;10:2781-8.

4. Albrecht E, Taffe P, Yersin B, Schoettker P, Decosterd I, Hugli O. Undertreatment of acute pain (oligoanalgesia) and medical practice variation in prehospital analgesia of adult trauma patients: a $10 \mathrm{yr}$ retrospective study. $\mathrm{Br} \mathrm{J}$ Anaesth. 2013;110:96-106.

5. Oberholzer N, Kaserer A, Albrecht R, et al. Factors influencing quality of pain management in a physician staffed helicopter emergency medical service. Anesth Analg. 2017;125:200-9.

6. Dißmann PD, Maignan M, Cloves PD, Parres B, Dickerson S, Eberhardt A. A review of the burden of trauma pain in emergency settings in Europe. Pain Ther. 2018. https://doi.org/10.1007/s40122-0180101-1.

7. Berben SAA, Schoonhoven L, Meijs THJM, van Vugt $A B$, van Grunsven PM. Prevalence and relief of pain in trauma patients in emergency medical services. Clin J Pain. 2011;27:587-92.

8. Eidenbenz D, Taffé P, Hugli O, Albrecht E, Pasquier M. A two-year retrospective review of the determinants of pre-hospital analgesia administration by alpine helicopter emergency medical physicians to patients with isolated limb injury. Anaesthesia. 2016;71:779-87.

9. Bredmose PP, Lockey DJ, Grier G, Watts B, Davies G. Pre-hospital use of ketamine for analgesia and procedural sedation. Emerg Med J. 2009;26:62-4. 
10. Entonox summary of product characteristics. http://www.mhra.gov.uk/spc-pil/?prodName=ENTO NOX\%20MEDICINAL\%20GAS\&subsName=NITRO US\%20OXIDE\&pageID=SecondLevel. Accessed 06 Sept 2018.

11. Savoia G, Coluzzi F, Di Maria C, et al. Italian Intersociety Recommendations on pain management in the emergency setting (SIAARTI, SIMEU, SIS 118, AISD, SIARED, SICUT, IRC). Minerva Anestesiol. 2015;81:205-25.

12. Penthrox ${ }^{\circledR}$ summary of product characteristics. https://www.medicines.org.uk/emc/medicine/313 91. Accessed 06 Sept 2018.

13. Blair HA, Frampton JE. Methoxyflurane: a review in trauma pain. Clin Drug Investig. 2016;36:1067-73.

14. Grindlay J, Babl FE. Review article: efficacy and safety of methoxyflurane analgesia in the emergency department and prehospital setting. Emerg Med Australas. 2009;21:4-11.

15. Coffey F, Wright J, Hartshorn S, et al. STOP!: a randomised, double-blind, placebo-controlled study of the efficacy and safety of methoxyflurane for the treatment of acute pain. Emerg Med J. 2014;31:613-8.

16. Coffey F, Dissmann P, Mirza K, Lomax M. Methoxyflurane analgesia in adult patients in the emergency department: a subgroup analysis of a randomized, double-blind, placebo-controlled study (STOP!). Adv Ther. 2016;33:2012-31.

17. Chin R, Maccaskill M, Browne G. A randomised control trial of inhaled methoxyflurane pain relief, in children with upper limb fracture. J Paediatr Child Health. 2002;38:A13.

18. Buntine $\mathrm{P}$, Thom $\mathrm{O}$, Babl F, Bailey M, Bernard S. Prehospital analgesia in adults using inhaled methoxyflurane. Emerg Med Australas. 2007;19:509-14.

19. Babl FE, Jamison SR, Spicer M, Bernard S. Inhaled methoxyflurane as a prehospital analgesic in children. Emerg Med Australas. 2006;18:404-10.

20. Oxer HF, Wilkes G. Methoxyflurane is a safe, easy, effective analgesic for prehospital pain relief. Prehosp Disast Med. 2007;22:s77.

21. Jephcott C, Grummet J, Nguyen N, Spruyt O. A review of the safety and efficacy of inhaled methoxyflurane as an analgesic for outpatient procedures. Br J Anaesth. 2018;120:1040-8.

22. Spruyt O, Westerman D, Milner A, Bressel M, Wein S. A randomised, double-blind, placebo-controlled study to assess the safety and efficacy of methoxyflurane for procedural pain of a bone marrow biopsy. BMJ Support Palliat Care. 2014;4:342-8.

23. Gaskell AL, Jephcott CG, Smithells JR, Sleigh JW. Self-administered methoxyflurane for procedural analgesia: experience in a tertiary Australasian centre. Anaesthesia. 2016;71:417-23.

24. Grummet J, Huang S, Konstantatos A, Frydenberg M. The 'green whistle': a novel method of analgesia for transrectal prostate biopsy. BJU Int. 2012;110(Suppl 4):85-8.

25. Huang S, Pepdjonovic L, Konstantatos A, Frydenberg M, Grummet J. Penthrox alone versus Penthrox plus periprostatic infiltration of local analgesia for analgesia in transrectal ultrasound-guided prostate biopsy. ANZ J Surg. 2016;86:139-42.

26. Lee C, Woo HH. Penthrox inhaler analgesia in transrectal ultrasound-guided prostate biopsy. ANZ J Surg. 2015;85:433-7.

27. Nguyen NQ, Toscano L, Lawrence M, et al. Patientcontrolled analgesia with inhaled methoxyflurane versus conventional endoscopist-provided sedation for colonoscopy: a randomized multicenter trial. Gastrointest Endosc. 2013;78:892-901.

28. Abdullah WA, Sheta SA, Nooh NS. Inhaled methoxyflurane (Penthrox) sedation for third molar extraction: a comparison to nitrous oxide sedation. Aust Dent J. 2011;56:296-301.

29. Windsor J, van der Kaaij J, Ellerton J, Oxer $H$, Hillebrandt D, Rodway G. Methoxyflurane as an analgesic for prehospital use at high altitude. High Alt Med Biol. 2009;10:201-2.

30. Mundipharma Pharmaceuticals srl. Efficacy and safety of methoxyflurane vaporized (PENTHROX ${ }^{\circledR}$ ) in the treatment of acute trauma pain in pre-hospital setting and in the emergency department in Italy: a multicentre, randomized, controlled, openlabel study. https://www.clinicaltrialsregister.eu/ ctr-search/trial/2017-001565-25/IT. EudraCT number 2017-001565-25. Accessed 06 Sept 2018.

31. Marinangeli F, Tomei M, Ursini ML, Ricotti V, Varrassi G. Helicopter emergency medical service in Italy: reality and perspectives. Air Med J. 2007;26:292-8.

32. Australian Government Department of Health Therapeutic Goods Administration. Database of adverse event notifications (DAEN). https://www. tga.gov.au/database-adverse-event-notifications-da en. Accessed 06 Sept 2018.

33. Cousins MJ, Mazze RI. Methoxyflurane nephrotoxicity. A study of dose response in man. JAMA. 1973;225:1611-6. 
34. Dayan AD. Analgesic use of inhaled methoxyflurane: evaluation of its potential nephrotoxicity. Hum Exp Toxicol. 2016;35:91-100.

35. Jacobs IG. Health effects of patients given methoxyflurane in the prehospital setting: a data linkage study. Open Emerg Med J. 2010;3:7-13.

36. Frangos J, Mikkonen A, Down C. Derivation of an occupational exposure limit for an inhalation analgesic methoxyflurane (Penthrox ${ }^{\circledR}$ ). Regul Toxicol Pharmacol. 2016;80:210-25.

37. Johnston S, Wilkes GJ, Thompson JA, Ziman M, Brightwell R. Inhaled methoxyflurane and intranasal fentanyl for prehospital management of visceral pain in an Australian ambulance service. Emerg Med J. 2011;28:57-63.

38. Oxer HF. Effects of Penthrox ${ }^{\circledR}$ (methoxyflurane) as an analgesic on cardiovascular and respiratory functions in the pre-hospital setting. J Mil Veterans Health. 2016;24:14-20.

39. Hjermstad MJ, Fayers PM, Haugen DF, et al. Studies comparing numerical rating scales, verbal rating scales, and visual analogue scales for assessment of pain intensity in adults: a systematic literature review. J Pain Symptom Manag. 2011;41:1073-93.

40. The College of Emergency Medicine. Management of pain in adults 2014. https://www.rcem.ac.uk/ docs/College\%20Guidelines/5w.\%20Management
\%20of\%20Pain\%20in\%20Adults\%20(Revised\%20 December\%202014).pdf. Accessed 06 Sept 2018.

41. Jensen MP, Karoly P. The measurement of clinical pain intensity: a comparison of six methods. Pain. 1986;27:117-26.

42. Ho K, Spence J, Murphy M. Review of pain measurement tools. Ann Emerg Med. 1996;27:427-31.

43. Karcioglu O, Topacoglu H, Dikme O, Dikme O. A systematic review of the pain scales in adults: which to use? Am J Emerg Med. 2018;36:707-14.

44. Borobia Pérez AM, Capilla Pueyo R, Casal Codesido JR, InMEDIATE Group, et al. Phase IIIb, open label randomised clinical trial to compare pain relief between methoxyflurane and standard of care for treating patients with trauma pain in Spanish emergency units (InMEDIATE): study protocol. IBJ Clin Pharmacol. 2017;1:e0008.

45. Medical Developments International Limited. A randomised, double-blind, multicentre, placebo controlled study to evaluate the safety and efficacy of methoxyflurane (PENTHROX ${ }^{\circledR}$ ) for the treatment of acute pain in children and adolescents from 6 to less than 18 years of age (presenting to an Emergency Department with minor trauma). https:// www.clinicaltrialsregister.eu/ctr-search/trial/2016004290-41/GB. EudraCT number 2016-004290-41. Accessed 06 Sept 2018. 